Killermann, Denkschr. Bay. Bot. Ges. Regensb. XV.pp. 7 et 11. tab. 1. fig. 3 (1922); Bourdot et Galzin, Hym. Fr. I. pp. 387 et 389.n. 619 (1927); Killermann, in Engler et Prantil, Nat. Pflanzenfam. Ed • 2. VI. p. 144 (1928).

Syn. Hymenochatella arida KARsten, Bidrag Finl. Nat. och Folk Finsk. Vet.-Soc. XLVIII. p. 428. n. 1184 (1889).

Hymenochcetella laxa KARSTEN, Bidrag Finl. Nat och Folk Finsk. Vet.-Soc. XLVIII. p. 429. n. 1185 (1889).

Hymenochate laxa KARSTEN in SACCARDO, Syll. Fung. IX. p. 228 (1891).

Corticium simulans BERKELEY et RAVENEI (1878).

Nomina Jap.: Shibu O-uroko-daké (mihi); Shibu Uroko-také (YASUDA in herb.).

HAB. Honshū : ad O-yama in pago Kadota-mura, Kita-aidzuGōri, prov. Iwashiro (Yamaura, mense Maii anno 1916); ad urbem Sendai, prov. Rikuzen (A. YAsudA, 24. Oct. 1920); in Gongen-mori prope urbem Sendai, prov. Rikuzen (A. YASUDA, 17. Oct. 1918); ad Dango-djima in monte Haku-san, prov. Kaga (A. YASUdA, 16. Ang. 1923); ad Shinowara, prov. Harima (U. OUve, 28. Maii 1919); adFutatsuishi, Nakakawahara, Tsuna-Gōri, insula Awadji (S. Matsuzawa, 3. Aug. 1919). Herb. Yasuda.

Distrib. Europa, America borealis.

\title{
Contributiones ad Cognitionem Florae Asiae Orientalis.
}

(continued from Vol. 43, p. 407.)

by

\section{G. Koidzumi.}

Hydrangea cuspidata (ThG.) MiqueI, in Annal. Mus. Bot. Lugd. Batav. III. (1867). 98;-Prol. Fl. Jap. (1867). p. 262;-KoIDz. in 'Tokyo Bot. Mag. XXXIX. (1925) p. 311.

Volkameria sp. BLUME, in Sched1, Herb. Lugd. Batav. No. 903. 257-943. quae est exempla Thunbergiana, et ad Royen distributa.

Nom. Jap. Niwa-azisai.

Hab. Japonia.

Species valde insignis foliis supremis longe cuspidatis subtus secus costas venasque setulis albis vestitis, et in axillis costae secundariae haud barbatis, basi late cuneatis, serraturis cuspidatis ; petiolis laxe 
setuliferis; inflorescentiae rachibus pedunculisque dense pilosis et setis longis albis laxe intermixtis, floribus ut videtur omnino neutralibus, sepalis extus secus venas pilosis; florum pedicellis pilosis.

The type specimen seems to be sterile garden-form, but a specimen in the Herbarium of Leiden, No. 903. 268-85, collected by THunBERG, which is "pro parte" of his Viburunm serratum, and determined as $H$. opuloides var. serrata by C. K. SCHNEIDER, is a normal fertile wild form.

This specimen is a small branch, but the essential characters agree very well to the type form; but THUnBERG did not mentioned the exact locality for this plant.

Folia elliptica apice brevicuspidata basi decurrenticuneata 6-7 $\mathrm{cm}$. longa, $3 \mathrm{~cm}$. lata, sursum serrato-dentata, media serrata, deorsum integra, supra tantum ad marginem setulosa, costa media supia dense crispato-puberula, corymbus $3 \mathrm{~cm}$. altus $5 \mathrm{~cm}$. latus, floribus pediceílisque glabris, calyce 5-dentato tubo turbinato dentibus acutis; fl. neutralibus 4, sepalis obovatis basi cuneatis extus laxe pilosis ut videtur rubicundis. Petala extus in alabastrum laxe albo-lineolata. Ovaria toto immersa, stylis 3 .

Hydranga acuminata S. et Z. Fl. Jap. I p. 110. t. 56. 57. (1840). var, setulifera $\mathrm{m}$.

Foliis subtus secus costas venasque pilis albis brevibus setulosis patentibus vestitis.

Nom. Jap. Oni-sawa-azisai.

Hab. Kiusiu: Nagasaki. (1g. Maxımowicz. 1863)

f. Buergeri (S. et Z.) $\mathrm{m}$.

H. acuminata var. Buergeri S. et Z. F1. Jap. I. p. 111. (1840)

$H$. Buergeri S. et $Z$. ibid. t. 57. (1840).

Foliis angustioribus lanceolatis.

Nom. Jap. Hosoba-sawa-azisai.

Hab. Japonia occidentalis.

Prunus persica STOKES.

f. versicolor (SIEB.) $\mathrm{m}$.

Persica vulgaris var. versicolor SifB. Catal. Rais. (1863) p. 36. Nom. Jap.

Hab. culta.

f. stellata (SiEb. et de VRIESE) $m$.

Amygdalus persica var. stellata SiEB. et de VrIEs. F1. Jardin II. (1859) 65. t. 6.

Persica stellata SIEB. Cata1. Rais. (1863) p. 36. 
Nom. Jap.

Hab. in hortis culta.

var. pendula (SIEB.) $\mathrm{m}$.

Persica pendula SieB. Catal. Rais. II. (1863) 36.

Nom. Jap.

Hab. in hortis culta.

Epimedium Sieboldianum n. sp.

Accranthus sagittatus. var. Miguki in schedl. Hb. Lugd. Batav. No. 898. 196-202.

The present species is very like to those of $E$. sagittatum in its flower construction, but the leaves, on the contrary very like to those of E. youngianum.

Planta florens $32 \mathrm{~cm}$. alta, rhizomate longo oblique ascendente atrobrunneo squamoso et radicante. Folia ad apices rhizomatis 3, biternata; petiolis $17-18 \mathrm{~cm}$. longis laevibus glabris, petiolis secundariis $4-7 \mathrm{~cm}$. longis ; foliolis cordato-ovatis acutis junioribus 2.8$4.0 \mathrm{~cm}$. longis, infra medium $16-25 \mathrm{~mm}$. latis, nascentibus dense brunneo-villosis, sed supra cito glabris, subtus pilis persistentioribus, margine setuliferis, basi profunde cordatis 7-9-nervis; petiolulis 1. -4.0 $\mathrm{cm}$. longis; ad foliorum nodum dense intricato-villosis. Scapus $30 \mathrm{~cm}$. altus laevis glaber, floribus albis racemosis, bracteis ovatis scariosis ; pedicellis erectopatentibus $10-14 \mathrm{~mm}$. longis molliter pilosis. Calyx phyllis 4 oblongis $4 \mathrm{~mm}$. longis apice obtusissimis minute laceratis deciduis. Petala oblonga $6 \mathrm{~mm}$. longa ecalcarata, stamina 4, exserta, filamentis $4 \mathrm{~mm}$. longis, antheris acutissimis $2.5 \mathrm{~mm}$. longis. Ovaria glabra $3 \mathrm{~mm}$. longa, stylis $2 \mathrm{~mm}$. longis.

Nom. Jap.

Hab. Japonia, lg. Siebold!

Ulmus propinqua KOIDZ. nom. aov.

Ulmus japonica (non SiEB.) SARGEN', Trees and Shrubs, II. (1913) p. 1;-C. K. SchN. in Oestr. Bot. Zeitschr. Bd. 66. (1916). s. 31.

Nom. Jap. Nire.

Hab. Japonia ubique distributa.

Sanicula elata Hamir. T. in Don. Prodr. Fl. Nepal. (1825) p. 183. Foliorum costa laevissima, serraturis apice longe cuspidatis.

var. japonica (SIEB $\mathrm{m}$.

Sanicula canadensis (non. L.) 'THG. F1. Jap. 116.

Myrrhis canadensis (non R. S.) SiEB. Verh. Batav. Genoot. XII. (1830) 384. 
Sanicula japonica SIEB. Syn. P1. Oec. 46. (1830).

Sanicula chinensis BunGE. Enum. (1831). 189.

Sanicula europaea (non L.) YABE Rev. Umbell. Jap. p. 19. (1902)

Sanicula europaea var. elata. MAK. Tok. Bot. Mag. XXII. (1908) p. 176. (excl. syn. Hamirit. Kurz. etc.).

The japanese plant differs from Indo-malayan species by having the costae of leaves scaberulous and less apiculate serration.

Nom. Jap. Umanomitsuba.

Hab. Japonia. Sp. typ. in Herb. Lugd. Batav. No. 908. 262-420.

Illicium japonicum SIEB. Syn. (1830) 50.

I. anisatum (non L.) THG. F1. Jap. (1784) 235.

I. religiosum S. et Z. F1. Jap. I. t. 1. (1835);-F1. Jap. Fam. Nat. 1. (1845) p. 185.

Nom. Jap. Shikimi.

Hab. Japonia australis.

Elaeocarpus Kobanmochi Kornz. nom. nov.

El. japonica SIEB. nud! Syn. (1830) 65. (quoad specim. Hb. Lugd. Batav. No. 908. 253-422.!)

El.japonica (non SieB.) S. et Z. F1. Jap. Fam. Nat. in Abhadl. Muench. Akad. IV. 2. (1845) p. 165.-REHD. et WILs. in P1. Wils. II. p. 360 .

Nom. Jap. Kobanmochi.

Hab. Japan. China.

Rosa Wichuraiana CREPIN, in Bull. Soc. Royal. Bot. Belg. XXV. (1866) p. 189 ;-KoIdz. in Tokyo Bot. Mag. XXXVI. (1923) p. 46.

var. poteriifolia (Fr. et SAV.) $\mathrm{m}$.

R. luciae var. poteriifolia Fr. et SAv. Enum. P1. Jap. II. (1879). p. 344 .

Foliola orbicularia vel obovalia minor $1 \mathrm{~cm}$. non excedentia, flores parvi solitari vel paucicymosi.

Nom. Jap. Hime-terihanoibara.

Hab. Japonia.

Deutzia Thunbergiana (MAx.) C. K. SchN. in Schedl. Hb. Lugd. Bstav. No. 904. 75-710.

D. scabra MAxim. var. Thunbergiana MAx. Rev. Hydr. 45.

"Filamenta non vel vix dentata, pili foliorm vix dissimiles (supra 5-6-, subtus 6-9-radiatis) non planis."

Sec. C. K. SCHNEIDER,

This is very remarkable for their veins which is pubescent under- 
neath, not steliato-pilose as in $D$. crenata.! Petals spathulate oblong, very narrow, acute. And the stellulate hairs of underface much longer than those of $D$. crenata and gives quite different appearance.

Wisteria brachybotrys S. et Z. F1. Jap. I. fasc. 5 (1839) p. 92.t. 45, (Icon. mala! descript. Optim.!)

Kraunhia floribunda var. brachybotrys (S. Z.) MAk. Tok. Bot. Mag. XXV. (1911) p. 18.

This is a distinct species, near to Wisteria venusta REHD. et WILS., but flowers are purpule according to SIEBOI,D, the anterior teeth of calyx not subulate, and calyx-tube not costate.

Foliola 4-6-jugata utrinque dense pubescentia, integra, petiolulata, stipellulata, inferiora ovata vel elliptico-ovata basi leviter cordata vel subtruncato-cordata raro rotundata, superiora oblonga, omnia acutata; petiolis tomentosis. Racemus 13-16 cm. longus, 20-22-florus, basi foliis plerumque binis vestitus; floribus purpureis, $2 \mathrm{~cm}$. longis in sicco; pedicellis $2.7 \mathrm{~cm}$. longis in sicco, tomentosis, bracteolis caducis, calyce pulvelulenti-tomentoso.

Nom. Jap. Yamafuji.

Hab. Kiusiu?

Morus integrifolia LEVEII. et VNT. in Bull. Acad. Intern. Geogr. Bot. Vol. XVII (1907). p. 111;=Cudrania triloba HANCE in Jour. Bot. VI. (1868). 49; Hook Icon. P1. t. 1792.

Nom. Jap. Hariguwa.

Hab. China.

I have seen this specimen in the Herb. Lugd. Batav. No. 913. 55293. The Originals of LEVEILLE.

Cryptomeria japonica DON. var. dacryoides (SIEB.) $\mathrm{m}$.

Cryptomeria dacryoides SIEB. in S. et Z. F1. Jap. II. fasc. 23. (1844). p. 53.

Nom. Jap.

Hab. in hortis culta.

Cephalotoxus Harringtonia (Forb.) Koch. Deiddrol. II. 2. (1873). s. 102 .

Taxus Harringtonia Forb. Pinet. Woburn. (1839) 217. t. 63.

Cephalotaxus pedunculata S. et Z. (nom. nud!) in Abhand. Akd. Muench. IV. 2. (1846). s. 232 ;-Endl. Syn. Conif. (1847). 238;-S. et Z. Fl. Jap. II. p. 67. t. 132. (1870). 
C. drupacea var. Harringtonia MiQ. apd PILGE R in ENGL. Pfl. Reich. Taxaceae s. 102.

Nom. Jap. Inukaya.

Hab. Japonia.

var. drupacea (S. et $Z$.)

Cephalotaxus drupacea S. et. Z. (nom. nud.!) 1.c. (1846) 232 ; apd Endu. Syn. Conif. (1847). 239;-S. et Z. F1. Jap. (1870). 66. t. 130. t. 131.

Taxus Inukaja KNIGH'T. Synop. Conif. (1850). 51.

Nom. Jap. Inukaya.

Hab. Japonia.

var. coraiana (SIEB.) $\mathrm{m}$.

C. drupacea var. fastigiata CARR. Conif. ed. 1. (1855) 465 ;-Rev. Hort. (1863). 349.

Podocarpus coraianus SIEB. in Annal. Soc. Hort. Pays.-Bas. (1844). p. 35. (nom. nud.!)

P. coraiana SIEB. in S. et Z. Fl. Jap. Fam. Nat. II. (1846). 232 (nom. nud.!)-apd EivdL. Syn. (1847). 217.

C. Harringtonia f. fastigiata KоCH, 1. c. (1873) 103.

C. Buergeri MiQ. Prol. F1. Jap. p. 333.

Nom. Jap. Chosenmaki.

Hab. in hortis culta.

Podocarpus macrophylla ('THG.) DON. var. Ma-kayi (Forb.) $\mathrm{m}$.

Taxus Makayi Forb. Pinet. Woburn (1839) 128.

Podocarpus macrpohyllus WALL. var. Maki S. et Z. in Jaarbk. Tuinb. (1844). p. 35. (nom. nud.!)-Abhand1. Akad. Muench. IV. 3. (1846) s. 232. (nom. nud.!)

Podocapus macrophylla Don. var. Maki S. et Z. apd. Endl. Syn. Conif. (1847). p. 216;-S. et. Z. F1. Jap. II. (1870) 71. t. 134.

P. Makoyi Bium. Rmphia. III. (1847). 215.

P. Maki SieB. et Zucc. Jaarbk. (1845) 67. (nom. nud.!)-(1848). 41. (nud.!)

P. macrophylla var. chinensis Max. in Mel. Biol. VII. (1870). 562.

P. chinesis Wali ex Blum. Rumph. III. (1847). 216.-Carr. Conif. (1867). 658.

P. macrophylla Ssp. Maki SIEB. apd PILGER in ENGL. Pfl. Reich, s. 80 .

Nom. Jap. Maki, Rakanmaki. 
Hab. in hortis culta.

Acer subtrilobum (KocH.) $\mathrm{m}$.

A. palmatum var. subtrilobum KocH. in Annal. Mus. Bot. Lugd. Batav. I. (1864) p. 251. (Koch. typ. Herb. Lugd. Batav. No. 908. 2681329),

A. trifidum (non TH'G.) Hr. et ARN. Bot: BEACHY: Voy. (1841) 174. et alius auct.!

A. Buergerianum MiQ. Annal. II. (1865) 88.

A. trinerve Dippeir. Handb. II. (1892) 428.

Nom. Jap. Toh-kaede.

Hab: China.

Acer Maximowiczianum MiqueL, in Archiv. Neerl. Sci. Exact. Nat: II. (1867). 472. 476.

Acer nikoense Maxim (non MiQ.* 1867) Mel. Biol. VI. (186i). 370., (excl. Syn. Negundo nikocnse MrQ.!)

Nom. Jap. Chohjanoki

Hab. Nippon borealis.

Juniperus utilis KOIDZ. nom. nov.

Juniperus rigida (non NoIs 1829). S. et Z. apd ENdL. Syn. Conif. (1847) p. 17 ;-S. et Z. Fl. Jap. II. (1870). p. 56, t. 125;-NAKAI Tok. Bot. Mag. XXXI. (1917) p. 23.

Nom. Jap. Nezu.

Hab. Japonia, Korea, Manshuria.

Acer circumlobatum Maxim. Mel Biol. VI. (1867). 368;-MiQ. in Archiv. Neerland. Sci. Exact. II. (1867) p. 475;-Max. Mei. Biol. X. (1880). p. 608;-PAx. Aceraceae in ENGL. Pfl.-Reich. (1902). s. 25.

Acer cirumlobatum, var. insulare Pax. in EngL. Bot. Jahrb. VII. (1886) s. 200.

A.japonicum THG. var. circumlobatum (MAx.) Kont. Rev. Acerac. Jap. (1911). p. 42. t. 25. fig. 4. 5.

This is a distinct species having the adult leaves quite glabrous on surface, sparingly villouse underface, with a distinct principal costa, costae densely adpressed-villouse undermeath.

Folia ambitu orbicularia vel obovalia, 11-circumlobata, lobis ovatis

*Acer nikoense Miq. Arch. Neer1. II. (1867). p. 472. 478.=Negundo nikoense Miqul. Annal. II. (1S65) p. 90.

Miquel typ. Hb. Lugd. Batav. No, 908. 259-1302.

This is 2 trifid leaves of Cissus Thunbergii S. et Z. (v. p. 110.) 
acutis incisis, petiolis adpresse villosis; fructibus iis $A$. japonicae valde simillimis alis subhorizontaliter patentibus.

Nom. Jap.

Hab. Shinano (lg. Tshonoske! 1864. Hb. Lugd. Batav. No. 908 268-1391. Specim. typic. authentic.!)

Actinidia rufa (S. et Z.) Planch. ex MiQ. Annal. Mus. Bot. Lugd. Batav. II. (1867). p. 15.

var. dulcissima Koinz. nov.

A. cordifolia MrQuer. ibid. (1867). p. 15.

Nom. Jap. Hiroha-nashikazura.

Hab. Kiusiu (lg. PIERoT! n. 445 in Sylva ad ripam fluvii Asiyagawa, prope Kojanose); Yakushima (lg. C. WRIGHr!)

Actinidia platyphylla A. Gray. apd. MiqueL in Annal. Mus. Bot. Lugd. Batav. III. (1867) p. 15.

A. rufa var. cordifolia Dunn. in Jour. Linn. Soc. XXXIX. (1911) p. 403. (excl Syn. Miq.)

A. volubilis (non PLANCH.) CARr. Rev. Hort. (1874)p. 395. fig. 54.

Nom. Jap. Kokuwadsuru.

Hab. Yeso, Honto.

Cimicifuga acerina (S. et $Z$.) $\mathrm{m}$.

Pitiosperma acerinum S. et Z. Abhandl. Akad. Muench. III. 3. (1843). s. 735. t. III. fig. g. et in Fl. Jaard. (1858). t. IV. fig. (1-11). (excl. spica et folium!) (excl. Syn. THG.!)

C. japonica var. acerina HUTH. in ENGL. Bot. Jalırb. XVI. (1893) s. 316;-Bull. Herb. Boiss. V. (1897) s. 1093.

Cimifuga japonica Mrover. Annal. III. (1867) p. 9. (sphalmate Cimifuga!)

Nom. Jap. Usubano-Mitsubashoma.

Hab. Nagasaki Desima (1g. Sif,Bold ! Hb. Lugd. Batav. No. 908. 181-1153. type!); Kiusiu (1g. MohnıkeI! Hb. Lugd. Batav. No. 908. 181-1154.); Kiusiu (1g. Sre Bot, ! Hb. Lugd. Batav. No. 908. 1811137.) ; Kiusiu (Siebold? Hb. Lugd. Batav. No. 908. 181-1148.); Kiusiu 1g. Buerger! Hb. Lugd. Batav. No. 908. 181-1158-1168-1143.); Kiusiu (SIEBold! No. 908. 235-459.)

Cimicifuga japonica (Thg.) Sprngr. Syst. Veget. II. (1825). 628. Actaea japonica THG. F1. Jap. (1874). 221.

Pitiosperma acerina S. et Z. F1. Jaard. (1858) t. VI. (excl. fig. 111) (tantum Spica et Folium!) 
Cimifuga biternata MiqueL (p. p.) in Annal. Mus. Bot. Lugd. Batav. III. (1867). p. 9.

Nom. Jap. Mitsubashoma.

Icon. Jap. Somokudsusetsu, ed. 1. vol. X. fol. 14.

Hab. Japonia australis.

var. biternata (S. et Z.) FINET et GAGN. Bull. Soc. Bot. Fr. LI. (1904). p. 521.

Pitiosperma biternata MIQ. Annal. III. (1867). p. 9.-Prol. F1. Jap. (1867). 197. (pro prate!)

Actaea biternata Mrouer. apd Huth. in EngL. Bot. Jahrb. XVI. (1893). 316,-Bull. Hb. Boiss. V. (1897). s. 1093.

Nom. Jap. Awabo, Midsufude.

Icon. Jap. Kwa-i, IV.t. 13.

Hab. Yedo (ex Hb. Sikbol.d! Hb. Lugd. Batav. No. 908. 181-407.)

Cimicifuga peltata (MAK.) $\mathrm{m}$.

C. japonica f. peltata MAK. Tok. Bot. Mag. XXIV. (1910) 140.

Nom. Jap. Ohba-shoma, Kikenshoma.

Icon. Jap. Somokudsusetsu, ed. 1. XVII. f. 16.

Hab. Japonia.

Cimicifuga (Pitiosperma) macrophylla n. sp.

Species insignis foliolis 3 amplis ultra $20 \mathrm{~cm}$ longis subtus ad costam dense pilosis, scapo simplice; florum phyllis intimis in ungue intranectariferis.

Herbae perennis circ. $70 \mathrm{~cm}$. alta, rhizomatibus crassis pauciramosis, radicantibus. Folia unica radicalia ternata petiolis $20-23 \mathrm{~cm}$. longis intus late sulcatis apice pubescentibus, basi stipulis 2 connatis spicae basi vaginantibus, petiolulis $10 \mathrm{~cm}$. longis ; foliolis membranaceis supra glabris subtus secus costas pilosis et ema basi pubescentibus, foliolis 1nediis ambitu obovalibus palmato-7-lobatis, $20 \mathrm{~cm}$. longis, omnibus basi profunde cordatis, lobis subinciso-dentatis, subito acuminatis dentibus mucronato-acutis. Scapus erectus simplex superne pubescens, spica 12-14 cm. longa dense pubescentia, floribus albis sessilibus. Perianthii tepala circ. 6 extimis oblongis interioribus orbicularibus intimis in ugue intus nectariferis. Stamina circ 28 filamentis inaequilongis clavato-carnosis antheris albis obtusis ellipticis basi sessilibus. Carpellum unicum ampulliato-oblongum brevistipitatum stylis latis brevibus stigma sessilia ampla. Cápsula oblonga $6 \mathrm{~mm} .1 \mathrm{~g} .3 \mathrm{~mm}$. lata transverse circ. 4-venosa $1 \frac{1}{2} \mathrm{~mm}$. longe stipitata, apice dorso stylifera; stylis persistentibus horizontaliter patentibus. Semina oblonga facie varie 
reticulata.

Nom. Jap. Tachi-shoma.

Hab. Nippon media (lg. Tschonoski! 1866. Hb. Lugd. Batav. No. 908. 181-1144.)

\section{Cimicifuga (Pitiosperma) chinensis n. sp.}

Praecedentim affinis sed foliis chartaceis supra lucidiusculis subtus albidis, binis minoribus; stipulis apice longioribus, scapo basi vaginato, apice pauciramoso, elatiore.

Herbae perennis, rhizomate crasso radicante. Folia 2 omnia radicalia, ternata, petiolis sulcatis $21-30 \mathrm{~cm}$. longis basi apiceque laxe pubescentibus; petiolulis mediis $13 \mathrm{~cm}$, extimis $11 \mathrm{~cm}$. longis; lamina media orbicularia $12 \mathrm{~cm}$. $1 \mathrm{~g}$. extima oblique ovata $17 \mathrm{~cm}$. longa, supra glabra subtus secus costas venasque dense pubescentia, basi profunde cordata, palmatim 7-9-1oba lobis acutatis inciso-dentatis. Scapus simplex apice pauciramosus pubescens, ramis omnibus floriferis erectis; floribus albis sessilibus. Perianthii tepalis 6 exterioribus obovatis interioribus orbicularibus, intimis basi intus nectario cyathiforme praeditis. Stamina circ. 30 inaequilonga. Carpella 2 glabra fere sessilia.

Hab. Western China. 1g. E. H. Wilson, n. 2509. (Hb. Lugd. Batav. No. 922. 312-720).

Albizzia Julibrissin Duraz. (1772). var. speciosa (THG.) $\mathrm{m}$.

Mimosa speciosa THg. Tr. Linn. Soc. II. (1794) 336.

Acacia Nemu Willdn. Sp. P1. IV. 2. (1805) 1065.

Albizzia. Nemu Benth. Lond. Jour. Bot. I. (1842). p. 527.

A typo recedit legumine utrinque acuto nec longe attenuato ut in typo.

Nom. Jap. Nemu-no-ki.

Hab. China, Japonia.

Dolichos Lablab L. Sp. P1. (1753) 725 ;-BANks Ic. Kaempf. (1791) p. 2 .

Lablab vulgaris SAvi. Diss. (1821). p. 19.

Dolichos ensiformis (non L.) THG. F1. Jap. (1784) p. 279;-BANKS Ic. Kaempf. (1791). t. 25.

Dolichos cultratus THG. Tr. Linn. Soc. II. (1794) 340;-WILLDN. Sp. P1. III. (1800) p. 1029.

Laíab cultratus DC. Prodr. II. (1825) 402 ,-Forb. et HEMSL. Jour. Linn. Soc. XXIII. 195;-S. et Z. Fl. Jap. Fam. Nat. I. (1845) s. 118.

Nom. Jap. Fujimame. 
Hab. in hortis eulta.

Canavalia ensiformis DC. Prodr. II.(1825). 404 ;-PIPER et DUNN in Kew Bull. (1922). 134.

Dolichos incurvus THG. F1. Jap. (1784). 280.

Canavalia incurva Dc. ibid. 404;-PIPER et DuNN ibid. 145;--S, et Z. F1. Jap. Fam. Nat. I. (1845) s. 118.

Nom. Jap. Natamame.

Hab. in hortis culta.

Desmodium caudatum (THG.) DC. Prodr. II. (1825) 337.

Hedysarum caudatum THG. F1. Jap. (1784) p. 286.

H. undatum THG. in schedl. Hb. Upsala.

H. laburnifolium PoIr. Encycl. Meth. Dict. Bot. VI. (1804). 422. (non SreB. ex DC.)

D. laburnifolium (POIR.) DC. Prodr. II. 337.

Nom. Jap. Misonaoshi.

Hab. Japonia australis.

Gleditschia japonica MrQUEL, in Annal. Mus. Bot. Lugd. Batav. III. (1867). p. 54.

G. horrida (THunB). S. et Z. F1. Jap. Fam. Nat. I. (1845). s. 121. in adnot. et s. 139, (non WILLDN. nec DESF.)

Fagara horrida Thunb. in Trans. Linn. Soc. Bot. II. (1794) p. 329.

Gleditschia horrida ('Thunb.) Makino in 'Tok. Bot. Mag. XVII. (1903). p. 12.

Nom. Jap. Saikachi.

Hab. Japonia australis.

\section{Agrimonia}

Clavis Specierum Japoniae

1, Foliola crassiuscula utrinque dense hirsuta subtus aureoglandulosa ; folia suprema trifoliolata; foliolis omnibus longe oblongis inter se aequilongis - Agrimonia japonica (MrQ.) Foliola subtus secus costas venasque laxius hirta, $\quad-2$

2, Foliola 5-3, supra pilosa subtus eglandulosa, obovata; folia suprema trifoliolata; foliolis lateralibus valde minoribus, stipulae semiovatae dentatae, - Agrimonia nipponica KoIDz. Foliola tenuia 5-7, folia radicalia 9-foliolata, omnia supra glabra subtus laxisissime argenteo-glanduligera, obovatocuneiformia versus basin integra cuneataque, apice acutata; stipulae integrae vel paucidentatae 
ovato-oblongae

- Agrimonia pilosa LEDEB.

Agrimonia japonica (MIQUEL) $\mathrm{m}$.

A. Blumei Don, (pro parte! quoad Plant. ex Japon.) General System Gard. Bot. II. (1832). p. 563;-MEver in Bull. Acad. St. Petersbg, vol. X. (1842) p. 563 ;-WALP. Repert. II. (1843) p. 42.

A. Eupatria (non Linn.) Thunb. Fl. Jap. (1784) p. 195;-KoIdz. Consp. Rosac. Jap. (1913) p. 210.

A. viscidula (non Bunge) S. et Z. F1. Jap. Fam. Nat. I. (1845) s. 125 ;-Fr. et SAv. Enum. Pl. Jap. I. (1875) p. 133.

A. suaveolens (non PURSH) BI. Bijdr. II. (1826) p. 1113. (pro parte).

Nom. Jap. Kimmidsuhiki.

Hab. Japonia.

This is very distinct from Agrimonia Eupatria LinN. by upwardly branching stem and hypanthium not rapidly reflexed and its tube acute obconical not depresso-campanulate. The present plant also somewhat near to Agrimonia striata Mrchx. of north America.

Agrimonia (Hemisphaeria) nipponica KoIDz. nov. sp.

The new species is closely allied to Agrimonia microcarpa WALLR. of north America, but is easily distinguishable by much ramose habit, and the leaves not canescent underneath.

Planta perennans circiter $80 \mathrm{~cm}$. alta, rhizomatibus lignosis àscendentibus, radice non tuberifero. Caulis versus basin longe hirsutus, versus apicem villosus superne pauci-pluriramosus ramis virgatis vel erecto patentibus spicatis. Folia interrupte pinnata inferiora 5-foliolata 12-13 cm. longa, superiora uniugata circiter $7 \mathrm{~cm}$ longa, suprema trifoliolata $3 \mathrm{~cm}$ longa, foliola obovato-elliptica vel obovata, apice obtusa vel rotundata, margine serrato-dentata, sessilia vel brevissime petiolulata, supra hirta subtus non glanduligera, praesertim secus venas longe hirsuta, foliolis appendicibus parvis tri- vel quinquedentatis sessilibus, foliolis lateralibus foliorum superiorum semper quam terminalia minoribus; stipulis semiovatis dentatis foliaceis. Spica laxiflora bracteis parvis trifidis, floribus parvis luteis, pedicellis brevibus pubescentibus. Calyx tubo obconico basi adpresse cano-hirsuto lobis ovatis acutis trinervis. Petala anguste oblonga apice rotundata. Hypanthium maturitate reflexum $4 \mathrm{~mm}$ longum, tubo hemisphaerico canohirsuto longitudinaliter elevato-venoso, margine spinis hamatis interioribus erectis, exterioribus erectopatentibus brevibus vestito.

Nom. Jap. Hime-kimmizuhiki. 
Hab. Yokohama. (lg. MAximowicz. 1862. Hb. Lugd. Batav. No. 908. 191-963. typ.,) ibid (No. 908. 191-1535.)

Agrimonia javanica Jungh. Java I (1852) s. 479-(1853) s. 664;Miq. Fl. Ind. Batav. I. (1855) 370.

A. Blumei Don. (p. p.!)

A. Eupatria (non L.) KooRd. Exkurs. H1. Jav. II. (1912) s. 333.

The present species is very remarkable in its tall much ramosed and very densely longhirsute stem; and also very narrow, relativeiy small and densely hirsute leaflets. Herbae perennes, caulibus elatis ramosis dense et longe ferrugineo-hirsutis. Folia interrupte pinnata foliolis 5-9 oblongo-oblanceolatis sessilibus versus bas n cuneatis utrinque densissime hirsutis, margine anguste longeque dentatis, rachidibus dense hirsutis, stipulis semiorbicularibus valde amplis grosse dentatis densissime hirsutis. Spica multiflora floribus pedicellatis, bracteis inferioribus foliaceis lobatis superioribus parvis trifidis. Hypanthia juniora non reflexa tubo obconico longitudinaliter elevato-venosa, spinis numerosis.

Nom. Jap.

Hab. Java: in montibus Tengger (1g. Junghun!) mt. Merbabu (JUNGHUN!)

Rubus (Corchorifolii) officinalis KoIDz. nov. sp.

Frutex, caule atro-purpurascente remote acureato valde flexuoso, ramulis acureis recurvis paucis armatis, glabris, florentibus brevibus simplicibus unifloris, foliis $2-4$ distantibus gerentibus, Folia $1,5-3,5 \mathrm{~cm}$ longe petiolata, ambitu subrotundata generaliter $3-7 \mathrm{~cm}$ longa ac lata, raro $8-13 \mathrm{~cm}$ longa ac lata, quinquepartita vel quinquesecta, lobis lateralibus ovatis usque ovato-lanceolatis, terminalibus lanceolatis vel subrhombeo-lanceolatis acuminatis rarius acutis, utrinque in costis pubescentibus, argute inciso-serratis, stipulis longe lanceolatis ad basin petioli adnatis acuminatis; petiolis aculeolatis; nloribus sigulis amplis $4-4,5 \mathrm{~cm}$ in diametro, pedicellis gracilibus glabris pendulis vel nutantibus; sepala ovato-oblonga obtusa mucronato-appendiculata utrinque dense pubescentia; petalis late elliptico-ovalibus albis circiter $17-20 \mathrm{~mm}$ longis margine repandis apice acute obtusis; ovaria aibo-tomentosa. Fructus ruber globoso-ovoideus $14 \mathrm{~mm}$ longus basi $15 \mathrm{~mm}$ latus dense pilosus, putamine rugoso.

Nom. Jap. Gosho-ichigo.

Hab. China: Fokien (DUnN No. 2626. Hb. Kew.) Kuatem mountains (M. de LATOUCHE Aprit 1898. Hb. Mus. Paris.) Chekiang (E. FABER No. 193. in anno 1889. Hb. Kew.) Tientai (E. FABER anno 1891 
Hb. Berol.); Hieou-ning (ZIKAWEI April 13, 1920. Hb. Mus. Paris.) Japonia: Kiusyu, prov. Buzen, Hikosan. (lg. KIyohara!)

\section{Rosa fujisanensis MAKINO.} var. setifera $n$. v.

Foliolis parvis 5-13 mm longis, subtus ad basim costae mediae interdum adpresse pilosae, petiolis pubescentibus. Racemus hemisphaericus cic. 6-florus abbreviatus, florum pedicellis circ. $5 \mathrm{~mm}$ longis dense glanduloso-setuliferis, rachidibus dense glanduloso-setosis spinosisque, spinis horizontaliter patentibus.

Nom. Jap.

Hab. Higo. Hyakkanishi; Kiusiu (Oldham! Hb. Lugd. Batav. No. 908. 198-956).

Rosa (Indicae) Sieboldii CREPIN (nom. nud!) FR. et SAY, Enum. P1. Jap. I. (1874). p. 136.

Rosa indica var. Sieboldii CRFP. in Schedl. Herb. Lugd. Batav. No. 908. 198-523-524.

Rosa pimpinellifolia L. var. MrQ. Annal. III. (1867). p. 39.

This is a distinct species, it differs from $R$. chinesis and $R$. odorata by closely branching habit with much shorter flowering branchlets, leaflets much smaller and somewhat glaucous beneath, the termial leaflet always obovato-elliptic, their apex suddenly acute, leaf-rachis aculeolate and glanduloso-setuliferous.

Ramis glabris aculeis recurvis basi dilatatis vestitis, ramulis florigeris 6-13 cm. longis 2-7-foliigeris crebre ramosis. Folia 5-7 rarissime 9-foliolata, 4-11 cm. $1 \mathrm{~g}$. rachiaibus glabris aculeolis recurvis vel rectiusculis setulis glanduliferisque sparse armatis, stipulis petiolo adnatis margine glanduloso-ciliolatis sed antice parte libero subulato glandulosociliolato; foliolis membranaceis utrinque glabris subtus glaucinis brevissime petiolulatis sursum serratis, terminalibus obovato-ellipticis apice subito acutis vel acutatis deorsum obtusis vel subcuneatis, maximis ad $4 \frac{1}{2} \mathrm{~cm} .1 \mathrm{~g} .2,4 \mathrm{~cm}$. latis longe petiolulatis, foliolis lateralibus superioribus ellipticis vel obovato-ellipticis subito acutis, inferioribus ellipticis acutis vel interdum obtusissimis. Flores pleni $2 \frac{1}{2}-5 \mathrm{~cm}$. in diametro rubri vel luteorosei, plerumque ad apices ramulorum solitarii raro gemini interdum trini, pedunculis $2-3 \mathrm{~cm}$. $1 \mathrm{~g}$. glaberrimi basi plerumque bracteis stipuliformibus lanceolatis acuminatis gladulosociliat is vestitis, calycis tubo ampulliforme glaberrimo, sepalis lanceolat is caudatcacuminatis extus superne tantum pilosis intus velutino tomentosis in anthesi reflexis integris, raro exterioribus paucilaciniatis. 
Styli dense pubescentes.

Nom. Jap.

$\mathrm{Hab}$, in Japonia culta. (Hb. Lugd. Batav. No. 908. 198-446.-423345-529.-546-546, 523. lg. Mohnike!) (529. $1 \mathrm{~g}$. Buerger !) (423. 446, $524,435,1 \mathrm{~g}$. SiEBOLD!)

Salomonia oblongifolia DC. Prodr. I. (1824). p. 344;-BTH. F1. Hongk. 44;-Hook. fil. Fl. Br. Ind. I. 207;-For B. et HEMsI. Jour. Linn. Soc. XXIII. p. 59.

S. stricta S. et Z. F1. Jap. Fam. Nat. I. (1845). s. 152.

Nom. Jap. Hinanokanzashi.

Distr. Japonia, Formosa, China, Malaya, India, Australia.

Schima Mertensiana (S. et $Z$. ) $\mathrm{m}$.

Cleyera Mertensiana S. et Z. F1. Jap. I. p. 154. (1841). F1. Jap. Fam. Nat. I. (1845). s. 164.

S. Noronhae MAx. Mel. Biol. XII. (1886). 426 (quoad P1. ex Bonin.)

S. Noronhae var. boninensis HAyA'TA.

S. boninensis NakaI. Tok. Bot. Mag. XXXII. (1918) 222.

Nom. Jap. Himetsubaki.

Hab. Bonin.

Corydalis Oldhamii n. sp.

C. Wilfoldii (non RGI.) MrQ. Annal. III. (1867) 13.

The present species is near to $C$. heterocarpa $\mathrm{S}$. et $\mathrm{Z}$., but readely distinguishable by always broader leaflets, not moniliform pods which always pendent and shorter; the caruncle of the seeds larger. And also distinct from $C$. Tashiroi MAK. by having muricate seeds.

Glaberrima circ. $6 \mathrm{dm}$. alta, ramosa. Folia longe petiolata latoovata vel ovata in ambitu, usque $18 \mathrm{~cm}$. longa $14 \mathrm{~cm}$. lata vel ultra, bi- vel tri-pinnatisecta, tenuia, subtus glanca; pinnis 3-2-jugatis petiolulatis; segmentis brevissime stipitatis obovatis orbiculari-obovatis vel ovatis; obtusissimis, basi lato-cuneatis, lobato-crenatis cum dentibus ovatis usque rotundatis apice mucronulatis, vel interdum lobulatis, saepe bi- vel trifidis, circ. ad $2.5 \mathrm{~cm} .1 \mathrm{~g} .2 \mathrm{~cm}$. latis; petiolis $10 \mathrm{~cm} .1 \mathrm{~g}$. vel ultra in inferioribus. Racemus terminalis et lateralis cum folio oppositus erectus pedunculatus, floribus luteis $13-15 \mathrm{~mm}$. longis, rachidibus $10-20 \mathrm{~cm}$. longis circiter 15-20-floratis; pedicellis $3-6 \mathrm{~mm}$ longis, bracteolis lanceolatis acuminatis quam pedicellus longioribus. Calcar saccatus 3-4 mm longus leviter curvato-descendens. Capsula vix laxe disposita cum pedicellis recurvis $6 \mathrm{~mm}$ longis semper pendens 
lanceolaia attenuata $2-2.5 \mathrm{~cm}$. longa, haud lomentaceo-constiricta, apice stylis persistentibus $4 \mathrm{~mm}$ longis stigmate distincto bilobo vesitita. Semina subreniformia atrata nitidiula dense minuteque muricata carunculo orbiculato pro maxima parte iecta.

Nom. Jap. Tsukushi-ohkikeman, Tsukushi-malubakikeman,

Hab. Nagasaki: lg. R. Oldham! 1862. m. 39. (Hb. Lugd. Batav. No. 908. 164-636.)

Clematis chinensis Retzius. Bot. Obsv. II. (1781). p. 18. t. 2 ;FORB. et HEMSL. Jour. Linn. Soc. XXIII. p. 3.

C. terniflora Dc. Syst. Veget. I. (1818) p. 137;-Max. Mel. Bio1. IX. 596;-Fr. et SAv. Enum. Pl. Jap. II. 262;-Huth Bull. Herb Boiss. (1897) p. 1061.

C. triternata (non DC.) S. et Z. F1. Jap. Fam. Nat. I. (1845) s. 176.

$C$. biternata (non DC.) S. et $Z$. in sched1. Hb. Lugd. Batav.

C. biternata (noi DC.) MrQ. Annal. III. (1867). p. 1.

C. flammula (non L) Morr. et DeCne. Bull. Acad. Brux. Belg. III. p. 173. (?) (1836).

Nom. Jap. Shina-senninso.

Hab. Japonia (Hb. Iugd. Batav. Nr. 908. 181-1056); Prov. Kii. (ex Huth 1. c.). Kiusiu, Loochoo, Formosa.

Haec species affinis $C$. paniculata THG. sed foliolis magis flaccidis, in sicco atratis, venis tenuioribus; infl. semper trichotomis, floribus atropurpureis sepalis extus pilosis duplo minoribus, ovario dense piloso nec longe villoso-tomentoso ut in $C$. paniculata.

Caulis glaber vel superne pilosus. Folia pinnatim 5 -foliolata ad 20 cm. longa, superne cirrhosa ; rachibus glabris; foilorum jugis distantibus, triangularibus vel ovato-triagularibus, membranaceis, supra parce pilosis subtus glabris, pedatinervis, costis subtus laevissime elevatulis, basi truncatis vel levissime cordatis, apice obtusis vel rotundatis et mucronatis, integerrimis, $2-6 \mathrm{~cm} .1 \mathrm{~g} .2-4 \mathrm{~cm}$. latis; sed foliorum superiorum foliolis elliptico-ovatis basi late brevicuneatis trinervis, petiolulis 1-2.5 cm. longis. Corymbus trichotome ramosus, cic. $11 \mathrm{~cm}$. latus sed superiorus tantun triflorus, rachibus laxe pilosis vel glabris; $2-5 \mathrm{~cm}$. vel ultra iongis bracteis semper bijugatis parvis subulatis, pedicellis supra basin bibracteolatis sed florum terminalium semper ebracteolatis nudis!, bracteolis parvis filifomibus, floribus $15 \mathrm{~mm}$. latis atropurpurascentibus, sepalis 4 lanceolato-oblongis acutis $7 \mathrm{~mm}$. $1 \mathrm{~g} .2 \mathrm{~mm}$. 1t. extus pilosis margine albo-tomentosis ; flamentis glabris, antheris linearibus $2 \frac{1}{2} \mathrm{~mm}$. $1 \mathrm{~g}$. Carpella circiter 8 , ovaria dense pilosa apice albo-comosa, stylis basi albo-villosis. 
Magnolis obovata THG. Trans. Linn. Soc. II. (1794). p. 336.

$M$. hypoleuca S. et Z. in Abhand1. Muench. Akad. IV. 2. (1845). s. 187.-Bot. Mag.t. 8077 .

$M$. hypoleuca S. et Z. var. concolor S. et Z. ibid. 187.

Nom. Jap. Hohnoki.

$\mathrm{Hab}$. Japonia.

I have seen the type of THUnBERG in Upsala, which is surely our "Hoonoki"!

Salix (Adenophylla) Chamissonis Anders. in DC. Prodr. XVII. 2, (1868) p. 290 ;-C. K. Schn. in Bot. Gazt. vol. 67, (1919) p. 63;-Jour. Arnol. Arbor. vol. I. p. 149; et vol. III. p. 73, 94, 95;-FIODERS in Arkif. Bot. Bd. 20, (1926) A. no. 6, p. 29;-Hulten in F1. Kamt. Il. (1928) p. 9.

Salix anglorum (non Cham.) Kuno, F1. Paramushir. (1922) p. 96. Nom. Jap.

Hab. Kurils : insula Paramushir.

Distr. Alaska, Chukchur, Kamschatca, ad Jakutsk provinciam.

Rubus (Corchorifolii) palmatoides O. KunTzE, Method. Spec. Beschreib. Rubus. (1879) p. 93.

Rubus simillis O. KUnTZE ibid. p. 94.

Rubus dulcis KoIDz. in Tokyo Bot. Mag. XXVII. (1925) p. 50 283. XXXIX (1925) p. 306.

Nom. Jap. Nagahano-momijiichigo.

Hab. Nippon ocidentalis, Sikok, Kyusiu.

I have seen KUNTZE's type in the herbarium Kew.

Astilbe micropnylla F. KNOLL. in Bull. Herb. Boiss. VII. (1907). s. 131 . fig. 3 .

A. chinensis Mig. in Sched. Hb. Mus. Bot. Lugd. Batav.

Species insignis, caulibus foliis radicalibusque laxe longe rufovillosis, folii rachidis nodo fasciculato-rufovilloso; foliolis sessilibus apice obtusis vel subrotundiobtusissimis, supra pilosis subtus ad costis pilis subdensis horizontalibusque vestitis!

Rhizoma lignosum. $80 \mathrm{~cm}$. alta, non ramosa; foliis triternatis, incisodentatis dentibus aristato-mucronatis; Infl. ramis $3-1 \mathrm{~cm}$ longis, erectopatentibus, dense pubescentibus pilis subulatis!, petalis spathulatis versus basim longe sensim angustatis.

Nom. Jap. Chidakesashi.

Hab. Yokohama. (Maxrmowicz! 1862, Hb. Lugd. Batav.) 
Parthenocissus Thunbergii (S. et Z.) NakaI in Makino's Jap. Jour. Bot. no. 56. (1930). p. (254.)

Cissus Thunbergii S. et Z. F1. Jap. Fam. Nat. I. (1845). s. 195.

Ampelopsis tricuspidata S. et Z. ibid. s. 196.

Vitis inconstans MIQ. Annal. I. (1863) p. 91.

Negundo? nikoense MrQ. Annal. II. (1865). p. 90,

Acer nikoense MiQ. Arch. Neerl. Sci. Exact. II. (1867) p. 472. 478. (non Max. 1867).

Quinaria tricuspidata Kon HNE. Dendrol. (1893). 398.

Ampelopsis Roylei KIRCH. Arb. Musc. (1864) 152.

Parthenocissus tricuspidata PlanCH. in DC. Monogr. V. (1887) 452.

Parthenocissus tricuspidata REHDER in Rhodora X. (1908). 29.

Nom. Jap. Natsu-zuta.

Hab. Japonia, Liukiu.

Decavenia (NAKAI) KoIDZ. nov. gen.

Pierostyrax sect. Decavenia, Nakar. Tok. Bot. Mag. (1924) XXXVIII. p. 3.

Flores hermaphroditi dichlamydei actinomorphi tetracyclici pentameri fere apopetali diplostemoni. Calyx dense stellulato-tomentosissimus campanulatus tubo ovario adnato 19-costato; dentes 5 ovati persistentes. Corollae petala 5 decidua calycis fauci affixa erectopatentia calycis dentibus alterna, in aestivatione imbricata, basi saepe subcohaerentia. Stamina 10 uniseriata e petalis sublibera subaequalia exserta; filamentis basi tantum cohaerentibus vel subliberis, villosis; antherae linearioblongae introrsae adnatae biloculares longitudinaliter dehiscentes. Gynoecium simplex, carpella plerumque 3 syncarpa, ovarium pro maxima parte inferum 3-4 raro 5-loculare multiovulatum; ovula in quoque loculo plura, medio axi affixa superioribus ascendentibus inferioribus pendulis; stylus elongatus subulatus apice indistincte 3lobus persistens. Drupa exsiccata cylindrica longe stipitata decemnervia dense hirsuta, apice stylo tomentoso persistente; semine albuminoso tenue, embryonibus axillaribus, cotyledonibus 2 quam radicula elongata brevioribus. Arbor ramulis juvenilibus saepe steilato-pubescestibus. Folia alterna petiolata simplicia decidua argute irregulariter minuteque denticulata juniora hinc inde pilis stellulatis vestita. Inflorescentia terminalis paniculata dense stellulato-tomentosa, floribus albis fragrantibus sessilibus; bracteis caducis.

Dacavenia micrantha (S. e.t Z.) $\mathrm{m}$.

Pterostyrax micranthum S.et Z.Fl. Jap. Fam. Nat. II.(1846).s. 132. 
Ramis novellis stellato-pubescentibus; foliis membranaceis oblongis acuminatis basi subrotundatis vel obtusissimis irregulariter minute denticulatis, utrinque laxe vel subtus crebre stellulato-pilosis, costa medio et venis II supra stellato-pubescentibus subtus stellato- et simpliciter pubescentibus; petiolis stellato-pubescentibus ; paniculis $6-8 \mathrm{~cm}$. longis racemoso-ramosis, ramulis $1-1.5 \mathrm{~cm}$. longis dense albo-stellulatotomentosis ; floribus sessilibus $6 \mathrm{~mm}$ longis dense stellulato-tomentosis.

Nom. Jap.

Hab. Japonia (1g. K. Itro! Hb. Lugd. Batav. No. 908. 240-298.)

Decavenia hispida (S. et $Z$.) $\mathrm{m}$.

Pterostyrax hispidum S. et Z. 1. c. 132.

Ramulis hornotinis laxe stellato-pubescentibus; foliis oblongis acuminatis basi obtusis supra laxe stellato-pilosis subtus pilis minutis stellulatis glaucino-velutino-tomentosissimis, venis supra pilis brevibus stellatis et longis simplicibus vestitis; fructibus dense gilvo-hispidis, stipitibus $2-5 \mathrm{~mm}$ longis.

Nom. Jap.

Hab. Japonia (SizBold! Hb. Lugd. Batav. No. 908. 240-296).

Decavenia japonica n. sp.

Foliis pracedente affinibus sed subtus non tomentosis tantum secus costas pubescentibus; fructibus longioribus stipitibus $4-7 \mathrm{~mm}$. longis.

Nom: Jap.

Hab. Fujiyama (1g. Tschonoski 1864!) Hb. Lugd. Batav. No. 908. 240-294.

Veronica virginiana L. Sp. P1. p. 13. (1753).

Inflorescentia ramosa ramis acuminatis erectis, foliis lanceolatis, floribus arcte sessilibus albis carneis vel pupureis.

Hab. America borealis.

var. sibirica (I) NAkAI. Tok. Bot. Mag. XXVI. (1912) 170.

$V$. sibirica L. Sp. P1. p. 12.

Inflorescentia simplex, rachibus pubescentibus, floribus sessilibus vel subsessilibus coeruleis vel albis; foliis oblongis breviacuminatis utrinque pilosis, caulibus glabris.

Nom. Jap. Yezo-kukaiso.

Hab. Sinano (Tschonoski 1864. Hb. Lugd. Batav. No. 908. 233451). Yeso. Sibiria.

var. japonica NAKAI 1. c. XXV. 1912. p. 170.

Inflorescentia simplex rarissime basi pluriramosa, ramis brevibus erecto-patentibus, rachibus pubescentibus, floribus longe pedicellatis 
coeruleis vel albis, foliis lanceolatis fere glabris.

Nom. Jap. Kukaiso.

Hab. Yeso. Nippon, Sikoku.

var. Zuccarinii m.

Veronica japonica.STEUD. Nom. ed 2.p. 757. (nom. nud.!) apd S. et Z. F1. Jap. Fam. II. (1846). s. 143.

Eustachya japonica RAFIN. ex STEUDEL, 1. c.

Toxodendra verticillata $\mathrm{S}$. et $Z$. in sched. Hb. Lugd. Batav. No. 908. 233-412.

Caulis pubescens. Inflorescentia simp!ex rachibus glabris, floribus coeruleis fere sessilibus vel sessilibus, foliis 3-5-7-verticillatis brevibus ellipticis vel obovato-ellipticis utrinque constrictis breviacuminatis plerumque dense pubescentibus.

Nom. Jap. Tsukushi-kukaiso.

Hab. Kiusiu: (Hb. Lugd. Batav, No. 908. 233-460-412,-402-422432-461.)

Hypericum (Homotaenium) Kinashianum n. sp.

Species perdistinctissima quoad fructus Hypericum oliganthum sat similis, sed sepalis nigro-striatis, folia late lineariaque, non pelucidopunctata.

Planta circiter $40 \mathrm{~cm}$ alta e basi ramosa, caule laeve, sub folia sine lineis decurrentibus. Folia linearia ad $4 \mathrm{~cm}$ longa, $9 \mathrm{~mm}$ lata, quam internodia longiora, membranacea supra atro-viridia subtus glancina, basi apiceque rotundata, utrinque nigro-punctata. Fructus orbiculariovoidea $6 \mathrm{~mm}$ lönga, sepalis linearibus, apice obtusis vel rotundatis inequilongis nigro-striatis.

Nom. Jap. Miyako-otogiri.

Hab. Japonia: prov. Yamashiro, Adagoyama (leg. N. KinAshi! 19, oct. 1921).

(to be continued) 